\title{
Quantum Yield Measurement of Fluorescent Zeolite Nanopigments
}

\author{
By Olivier Nicolet, Stefan Huber, Caroline Lovey, Sabrina Chappellet, \\ Juliette Perrenoud, Marc Pauchard, Rolando Ferrini,* and Libero Zuppiroli
}

Organic fluorescent molecules are infiltrated in the channels of zeolite $\mathbf{L}$ nanocrystals, thus creating organic-inorganic fluorescent nanoparticles. Combined with dielectric matrices, these fluorescent nanopigments open the way to the realization of novel optical devices. In this paper, the optical measurement of the quantum yield of fluorescent zeolites by means of a precise and reliable diffuse reflectance technique is presented. Several possible factors that may affect the fluorescence quantum yield are also investigated.

or sol-gel matrix, ${ }^{[6-8]}$ it is possible to retain fluorescent materials with dye concentrations up to $0.75 \mathrm{M}^{[2]}$ In particular, the high concentration of monomer fluorescent dyes and the anisotropic arrangement of their dipole moments in zeolite hosts have led to the fabrication of interesting lasing materials. ${ }^{[9,10]}$ It has also been demonstrated that, by organizing cationic fluorescent dyes in the linear channels of zeolite L nanocrystals, an artificial antenna system for light harvesting and fast anisotropic transport can be realized. ${ }^{[2]}$ Moreover,

\section{Introduction}

In recent years, besides their widespread commercial use as catalysts and ion-exchangers, ${ }^{[1]}$ nanoporous zeolites have been demonstrated to be a very versatile inorganic host for the supramolecular organization of organic dye molecules, complexes, and clusters. ${ }^{[2]}$ In this paper, zeolite L, which consists of cylindrically shaped aluminosilicate porous nanoparticles with one-dimensional channels running along the crystal axis, is considered. These channels can be filled with organic guest molecules in order to create hybrid organic-inorganic systems. ${ }^{[2]}$ Synthesis procedures have been established to embed different organic molecules into zeolite L and to study several photochemical and photochromic processes. ${ }^{[3,4]}$ In particular, the geometrical constraints of the host framework lead to the supramolecular organization of the guest molecules into the channels. ${ }^{[5]}$ For instance, including fluorescent dyes into zeolite L has enabled the fabrication of new fluorescent pigments. ${ }^{\left[{ }^{[2]}\right.} \mathrm{By}$ exploiting the energy transfer between two or more combined fluorescent dyes, a large variety of colors can be obtained. Moreover, due to the limited space available in the zeolite channels (i.e., minimal diameter 7.1 formation, and unsolicited bimolecular reactions can be inhibited and very high concentrations of monomer dye molecules can be obtained. ${ }^{[2-4]}$ Therefore, unlike with dyes dispersed in a polymer

[*] Dr. R. Ferrini, Dr. O. Nicolet, Dr. S. Huber, Prof. Dr. L. Zuppiroli Laboratoire d'Optoélectronique des Matériaux Moléculaires (LOMM) Ecole Polytechnique Fédérale de Lausanne (EPFL)

$\mathrm{CH}-1015$ Lausanne (Switzerland)

E-mail: rolando.ferrini@epfl.ch

C. Lovey, Dr. S. Chappellet, Dr. J. Perrenoud, Dr. M. Pauchard

Functional Layers, ILFORD Imaging Switzerland $\mathrm{GmbH}$

$\mathrm{CH}-1723$ Marly (Switzerland)

DOI: 10.1002/adfm.200801421 infiltrating organic dyes into the channels of zeolite $\mathrm{L}$ can protect them against chemical attack, photobleaching, and thermal decomposition. ${ }^{[3,11,12]}$ Other functionalities can be added by functionalizing the external surface of the dye-loaded zeolite $\mathrm{L}$ and by embedding it into a polymer matrix. ${ }^{[13,14]}$ This opens the way to the use of fluorescent zeolite pigments for the fabrication of, for example, lenses, absorbing and fluorescent filters, polarizers, windows, optical data storage and light emitting devices, light harvesting materials, and fluorescence concentrators. ${ }^{[13,15-17]}$

Knowing the quantum yield of the zeolite nanoparticles is of paramount importance for the realization and the optimization of the optical devices mentioned above. Nevertheless, in spite of the intense research effort on zeolite hybrid pigments, little can be found in the literature on this fundamental property. To our knowledge, a quantitative study of the quantum yield of zeolites infiltrated with different classes of fluorescent dyes is still lacking. In particular, we observe that, even if the encapsulation into zeolite L may contribute to improve the stability of the organic dye (compared to its stability in non-deoxygenate solutions), ${ }^{[11,18]}$ the host-guest interactions can also affect the fluorescence quantum yield. ${ }^{[2]}$ For instance, the latter may strongly depend both on the loading efficiency of the organic dyes and on the physicochemical properties of the surrounding framework, such as its polarity and acidity, or the steric confinement of the dye molecules. Therefore, although the optical properties and the quantum yield of most fluorescent dyes are reported in the literature ${ }^{[19]}$ or can be easily measured in solution, ${ }^{[20,21]}$ a systematic and quantitative study of the quantum yield of fluorescent zeolite pigments is necessary. In this paper, we measure the quantum yield of zeolite L loaded with fluorescent perylene molecules by means of a diffused reflectance technique. The materials and their optical characterization are presented in Section 2. The diffused reflectance measurements ${ }^{22-25}$ are discussed in Section 3, where several factors that may influence 


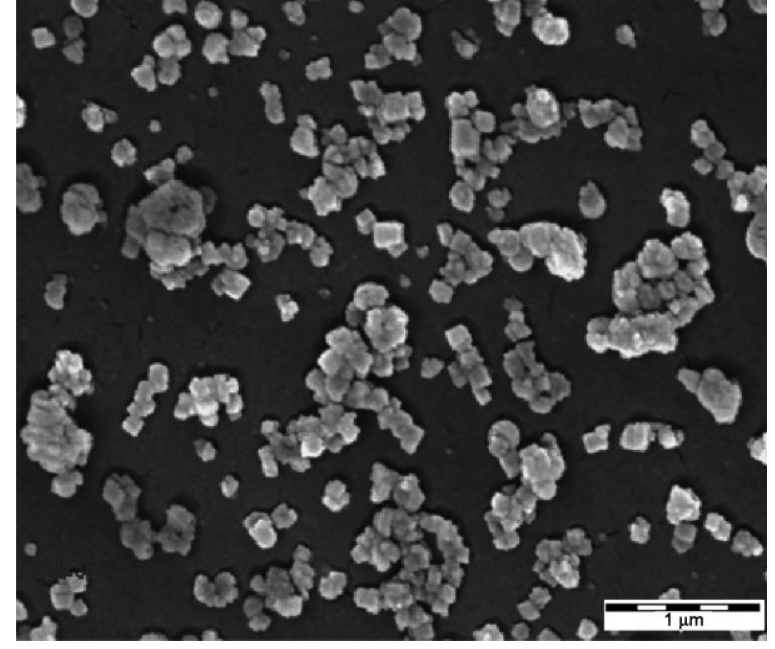

Figure 1. Scanning electron microscopy image of $200-n m$ zeolite L crystals synthesized using a hydrothermal process.

the zeolite quantum yield are investigated. Conclusions and perspectives are illustrated in Section 4. In Section 5, details are given both on the pigment and the sample preparation, and on the optical measurements.

\section{Materials}

We studied hybrid fluorescent pigments consisting of zeolite L (inorganic host) loaded with fluorescent perylene molecules (organic guest) that are well known for their high quantum yield and good stability. Zeolite L nanoparticles with an average size in the order of $200 \mathrm{~nm}$ were used (see Fig. 1). 3,9-Perylenedicarboxylic acid diisobutyl ester (PDB; see the inset in Fig. 2A) was chosen as the model fluorescent molecule. We remark that PDB molecules have the right physico-chemical properties to be infiltrated into the zeolite L channels by a gas-phase loading method (see the Experimental Section). ${ }^{[3,26]}$ Moreover, we highlight that it has already been demonstrated that molecules even larger than PDB can be infiltrated by this method into the $7.1 \AA$ channels of zeolite $\mathrm{L}$ when the right loading conditions are chosen. ${ }^{[27]}$

The measured steady-state excitation (dotted line) and emission (solid line) spectra of PDB molecules highly diluted in toluene $\left(10^{-6} \mathrm{M}\right)$ are shown in Figure 2A. For the excitation spectrum, the emission was collected at $560 \mathrm{~nm}$, while, for the emission spectrum, the excitation was performed at $440 \mathrm{~nm}$. We remark that the shoulder appearing at $560 \mathrm{~nm}$ in the emission spectrum is due to the formation of dimers in the solution.

The PDB molecules were infiltrated into the zeolite channels using the gas-phase loading method. ${ }^{[3,26]}$ The actual infiltration of perylene molecules into the zeolite channels was experimentally assessed, as it is discussed in detail in the Experimental Section. Moreover, co-adsorption of cationic molecules at the channel entrances was used to stabilize the zeolite nanopigments, thus preventing the infiltrated PDB molecules from leaking out of the channels after the loading procedure (see Experimental Section).

The percentage of the adsorption sites occupied by the PDB molecules (i.e., the loading efficiency) was measured
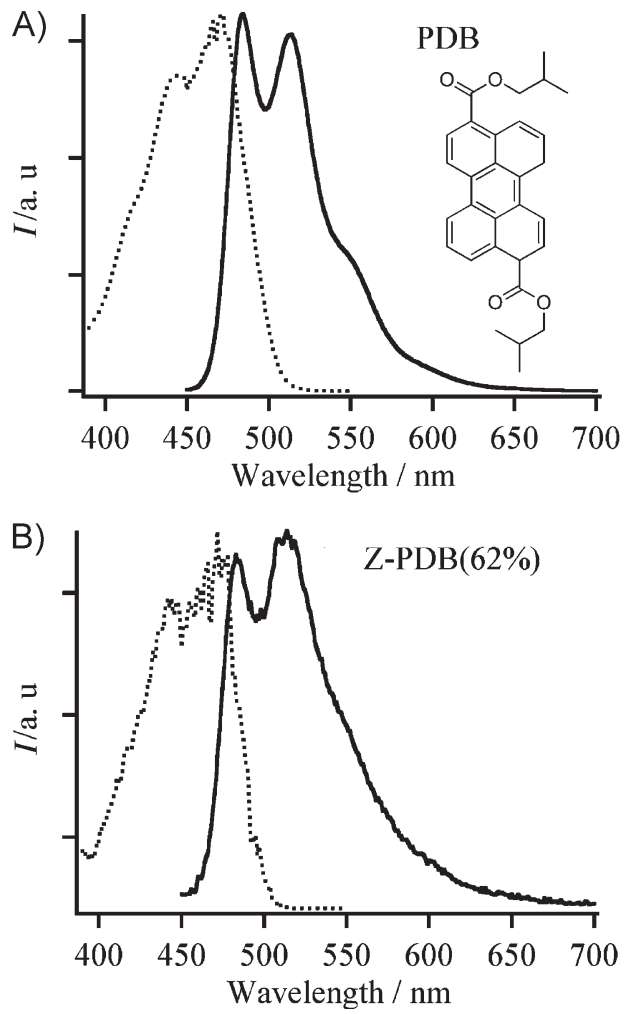

Figure 2. Steady-state excitation (dotted line) and emission (solid line) spectra of A) PDB molecules and B) Z-PDB nanoparticles dispersed in toluene (concentration $=10^{-6} \mathrm{M}$ and $5 \mathrm{mg} \mathrm{L}^{-1}$ for PDB and Z-PDB, respectively). For the excitation spectrum, the emission was collected at $560 \mathrm{~nm}$, while, for the emission spectrum, the excitation was performed at $440 \mathrm{~nm}$. The structure of the PDB molecule is shown in the inset of $(A)$.

(see Experimental Section for details). Several PDB-loaded zeolite pigments (Z-PDB) were obtained with loading efficiencies of 12 , 32,62 , and $69 \%$. The steady-state excitation and emission spectra of the Z-PDB nanoparticles dispersed in a toluene solution (content $=5 \mathrm{mg} \mathrm{L}^{-1}$ ) were measured in the same conditions as for the PDB molecules (see Fig. 2B). We highlight that, independently from the loading efficiency, the Z-PDB emission spectrum well agrees with the PDB spectrum in the main emission region, while the dimer-related shoulder at $560 \mathrm{~nm}$ becomes negligible, thus confirming that the infiltration of organic molecules into the zeolite nanochannels prevents them from aggregation.

In order to measure the quantum yield of both the PDB molecules and the Z-PDB pigments, they were adsorbed onto microcrystalline cellulose samples, ${ }^{[23]}$ which were prepared using a solvent evaporation method (see the Experimental Section). This substrate was chosen both for its high diffuse reflectance (i.e., $\geq 0.80$ ) in the visible spectral region and for its porosity that allows a good dispersion of fluorescent molecules and nanoparticles. The measured emission spectra of PDB and Z-PDB (62\%) adsorbed onto the cellulose are shown in Figure 3A and B, respectively, for an increasing fluorophor content (the fluorophor to cellulose weight proportions were chosen to guarantee fluorophor absorption values in the order of $1-15 \%$ at the excitation wavelength). In the insets, the emission spectra for the 

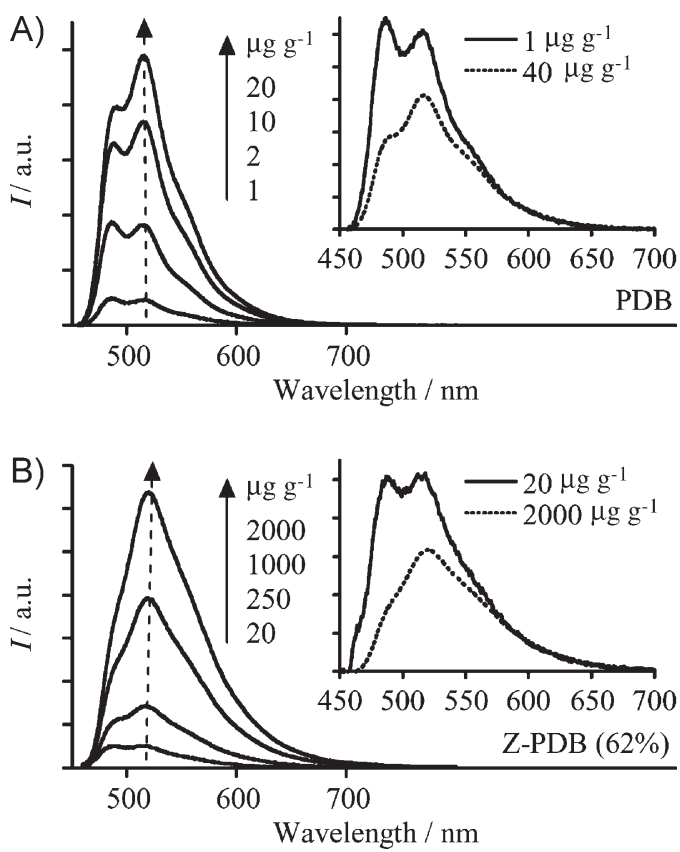

Figure 3. Steady-state emission spectra of A) PDB molecules and B) ZPDB (62\%) nanoparticles adsorbed onto microcrystalline cellulose for increasing fluorophor contents [in $\mu \mathrm{g}$ (fluorophor) $\mathrm{g}^{-1}$ (cellulose)]. In the insets, the spectra for the lowest and the largest fluorophor concentrations [1 (solid line)-40 (dotted line) $\mu \mathrm{g} \mathrm{g}^{-1}$ and 20 (solid line)-2000 (dotted line) $\mu \mathrm{g} \mathrm{g}^{-1}$ for PDB and Z-PDB (62\%), respectively] have been normalized at $600 \mathrm{~nm}$ (where self-absorption is absent: see Fig. 2) for a better comparison of their spectral shape.

lowest and the largest fluorophor concentrations [1 (solid line)-40 (dotted line) $\mu \mathrm{g} \mathrm{g}^{-1}$ and 20 (solid line)-2000 (dotted line) $\mu \mathrm{g} \mathrm{g}^{-1}$ for PDB and Z-PDB (62\%), respectively] are normalized at $600 \mathrm{~nm}$ (where self-absorption is absent: see Fig. 2) for a better comparison of their spectral shape. We observe that, for the lowest fluorophor dispersion values, the PDB and Z-PDB spectra well agree with the corresponding spectra measured in solution (see Fig. 2). However, for the highest dispersion values, the emission spectra are strongly affected by the increasing fluorophor concentration. Note that, on one hand, while for ZPDB $(62 \%)$ the emission intensity linearly increases when increasing the fluorophor content, for PDB the linearity is lost for the largest molecule concentrations. The latter difference can be indeed taken as an indirect evidence of the encapsulation of PDB molecules into the zeolite channels in Z-PDB (see Experimental Section). On the other hand, since for both PDB and Z-PDB (62\%) the Stokes shift is small (see Fig. 2), the spectral shape changes due to self-absorption effects (see Experimental Section).

\section{Diffuse Reflectance Measurements: Results and Discussion}

Diffuse reflectance measurements ${ }^{[22-24]}$ were used to measure the quantum yield of both PDB molecules and Z-PDB nanoparticles adsorbed on the cellulose. In particular, an integrating sphere was used to measure the diffuse reflectance of microcrystalline cellulose substrates containing either fluor-
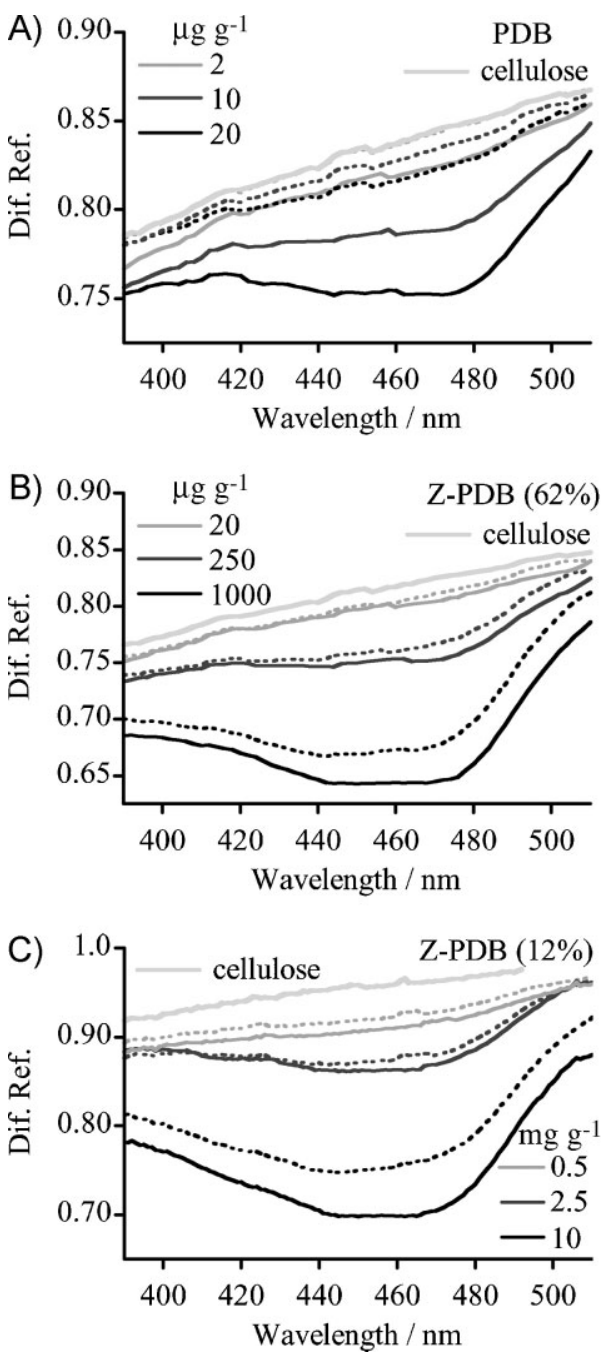

Figure 4. Diffuse reflectance spectra measured with ( $R_{\mathrm{f}}$ : solid line) and without ( $R_{\text {wof: }}$ dotted line) a cut-on filter in front of the detector for the bare cellulose substrate ( $R_{\text {sub }}$ : light gray line) and substrates containing A) PDB, B) Z-PDB (62\%), and C) Z-PDB (12\%), respectively, for different fluorophor concentrations: $\mathrm{PDB}=2,10$, and $20 \mu \mathrm{g}$ (fluorophor) $\mathrm{g}^{-1}$ (cellulose); $\mathrm{Z}-\mathrm{PDB}(62 \%)=20, \quad 250$, and $1000 \mu \mathrm{g}$ (fluorophor) $\mathrm{g}^{-1}$ (cellulose); $\mathrm{Z}-\operatorname{PDB}(12 \%)=0.5,2.5$, and $10 \mathrm{mg}$ (fluorophor) $\mathrm{g}^{-1}$ (cellulose).

escent molecules (e.g., PDB) or Z-PDB nanoparticles with or without a cut-on filter in front of the detector. ${ }^{[25]}$ This filter was chosen to prevent the fluorescence emission from being detected.

Diffuse reflectance spectra of microcrystalline cellulose substrates containing PDB molecules, Z-PDB(62\%) or Z$\operatorname{PDB}(12 \%)$ nanoparticles were measured separately for several increasing fluorophor concentrations. The spectra measured with ( $R_{f}$ : solid line) and without $\left(R_{\text {wof: }}\right.$ dotted line) the cut-on filter for the bare cellulose substrate $\left(R_{\text {sub: }}\right.$ : light gray line) and the substrates containing PDB, Z-PDB (62\%), and Z-PDB (12\%) are shown in Figure $4 \mathrm{~A}-\mathrm{C}$, respectively, for different fluorophor contents in the cellulose: PDB $=2,10$, and $20 \mu \mathrm{g} \mathrm{g}^{-1}$; Z-PDB $(62 \%)=20,250$, and $1000 \mu \mathrm{g} \mathrm{g}^{-1}$; Z-PDB $(12 \%)=0.5,2.5$, and $10 \mathrm{mg} \mathrm{g}^{-1}$. The decrease in the diffuse reflectance signal of the doped substrates with respect to the bare cellulose is due to the 
absorption of the fluorophors between 400 and $500 \mathrm{~nm}$ (see Fig. 2). This decrease is stronger when the cut-on filter is used (solid lines) since the latter prevents the fluoresced photons from being detected. We observe that the difference between the diffuse reflectance signal with and without the cut-on filter is proportional to the fluorophor quantum yield: the higher the fluorescence efficiency, the larger this difference is, that is, qualitatively, the measured quantum yield is much lower for ZPDB pigments than for PDB molecules. We highlight that, since for all the considered fluorophors this difference increases linearly with the increasing fluorophor content and the same linear behavior is found for Z-PDB pigments with low and high dye loading, our optical technique i) is not affected by the refractive index contrast between the fluorophor and the matrix (i.e., it takes intrinsically into account the light scattering) and ii) it enables reliable quantum yield measurements of zeolite nanopigments independently from the dye content into the channels. Note that for Z-PDB (12\%) the diffused reflectance signal is much lower than for Z-PDB (62\%) and a larger fluorophor content into the cellulose (almost one order of magnitude) had to be used to perform the optical measurements. This confirms qualitatively that the dye content in Z-PDB (12\%) is much lower than in Z-PDB (62\%) (see Experimental Section) and that our experiments were performed in the region where the Z-PDB emission intensity strongly increases with the dye loading.

In order to measure the quantum yield, the diffuse reflectance values $R_{\mathrm{f}}, R_{\mathrm{wof}}$, and $R_{\mathrm{sub}}$ at the absorption wavelength $\left(\lambda_{\mathrm{Abs}}=460 \mathrm{~nm}\right)$ were used to calculate both the fluorescence intensity $\left(I_{\text {Fluo }}\right)$ and the intensity of the excitation light absorbed by the fluorophor $\left(I_{\mathrm{Abs}}\right)$. These quantities were obtained using the equations by Ferreira et al. ${ }^{[23]}$ that take into account the spectral response of the detector and a small residual transmission through the cut-on filter (see Equations 1-3 in the Experimental Section). In Figure $5, I_{\text {Fluo }}$ is plotted as function of $I_{\mathrm{Abs}}$ for different concentrations of PDB molecules (gray circles, dotted line), Z-PDB (62\%) (black circles, dotted line), and Z-PDB (12\%) (black squares, dashed line) nanoparticles adsorbed onto

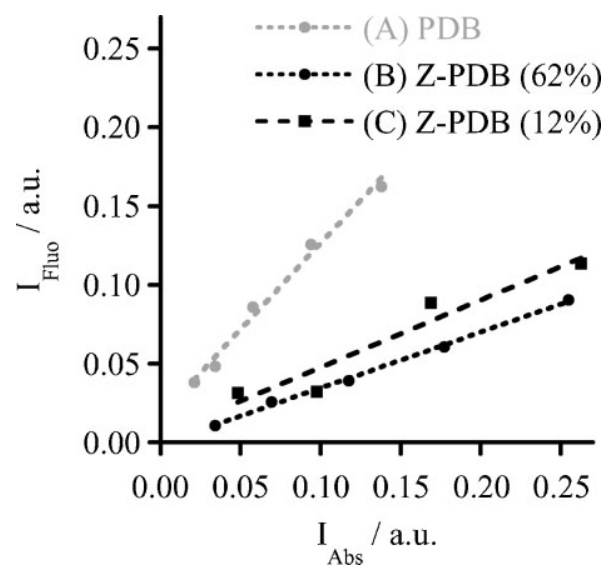

Figure 5. Fluorescence intensity $\left[I_{\text {Fluo }}=\left(R_{\text {wof }}-R_{\mathrm{f}}\right) /\left(f_{S}-f_{S, T}\left(1-R_{\text {wof }}\right) /\right.\right.$ $\left.\left.\left(1-R_{f}\right)\right)\right]$ as a function of the intensity of the excitation light absorbed by the fluorophor $\left[I_{\mathrm{Abs}}=R_{\mathrm{sub}}-R_{\mathrm{f}}\right]$ for different concentrations of A) PDB molecules (gray circles, dotted line), B) Z-PDB (62\%) (black circles, dotted line), and C) Z-PDB (12\%) (black squares, dashed line) nanoparticles adsorbed onto the cellulose substrates.
Table 1. Measured quantum yield values for PDB molecules and Z-PDB nanoparticles adsorbed onto microcrystalline cellulose substrates [the quantity of loaded PDB molecules, as well as the concentration in solution, corresponding to the indicated loading efficiencies (\%) are given in the footnotes]. $\phi$ and $\phi_{\text {cor }}$ are the values obtained without and with the selfabsorption correction, respectively. The quantum yield value of PDB molecules measured in ethanol is 0.9 [28].

\begin{tabular}{lcc}
\hline Fluorophor & $\phi$ & $\phi_{\text {Cor }}$ \\
\hline PDB & 0.93 & $0.95 \pm 0.07$ \\
Z-PDB(12\%) [a] & 0.28 & $0.45 \pm 0.05$ \\
Z-PDB(32\%) [b] & 0.38 & $0.48 \pm 0.05$ \\
Z-PDB(62\%) [c] & 0.28 & $0.37 \pm 0.04$ \\
Z-PDB(69\%) [d] & 0.35 & $0.44 \pm 0.04$ \\
\hline
\end{tabular}

[a] $6.8 \mathrm{mg}$ (PDB) $\mathrm{g}^{-1}$ (zeolite); $0.03 \mathrm{~mol} \mathrm{~L}^{-1}$. [b] $18.1 \mathrm{mg}$ (PDB) $\mathrm{g}^{-1}$ (zeolite); $0.09 \mathrm{~mol} \mathrm{~L}^{-1}$. [c] $35.0 \mathrm{mg}$ (PDB) g $\mathrm{g}^{-1}$ (zeolite); $0.17 \mathrm{~mol} \mathrm{~L}^{-1}$. [d] $\left.38.9 \mathrm{mg}^{(P D B}\right) \mathrm{g}^{-1}$ (zeolite); $0.19 \mathrm{~mol} \mathrm{~L}^{-1}$.

the cellulose substrates. $I_{\text {Fluo }}$ increases linearly with $I_{\text {Abs }}$ as the fluorophor concentration increases. We remark that, even at the highest concentration values, no deviation from linearity is observed. The quantum yield value ( $\left.\phi_{\text {measured }}\right)$ corresponds to the slope of the linear fit of $I_{\text {Fluo }}$ as a function of $I_{\mathrm{Abs}}$. Two additional corrections had to be introduced to take into account i) the large residual transmission through the cut-on filter ${ }^{[25]}$ and ii) selfabsorption effects (see the Experimental Section). The final corrected quantum yield values ( $\phi_{\text {Cor }}$ ) for PDB, Z-PDB $(62 \%)$ and Z-PDB (12\%) are $0.95 \pm 0.07,0.37 \pm 0.04$, and $0.45 \pm 0.05$, respectively (see Table 1 ). On one hand, the quantum yield of PDB molecules directly adsorbed onto the cellulose substrates is found to be higher than 0.90 . This latter is the value for nondeoxygenate PDB solutions in ethanol measured using a standard procedure and taking a fluorescein solution $(0.1 \mathrm{M} \mathrm{NaOH})$ as reference. ${ }^{[28,29]}$ On the other hand, the quantum yield is more than halved once the PDB molecules have been infiltrated into the zeolite nanoparticles. This may be due to several factors: i) the high concentration of the PDB molecules inside the zeolite channels, ii) the fluorescence quenching due to dimer formation at the outer surface of the zeolites, iii) the physico-chemical properties of the channel environment. These factors were investigated separately and are discussed in details in the following.

First of all, the influence of the zeolite loading on the Z-PDB quantum yield was studied. The quantum yield values measured for several Z-PDB nanoparticles are shown in Table 1 as a function of the measured PDB loading. We remark that, independently from the loading value, an average quantum yield of 0.40 was obtained. Therefore, the decrease of the fluorescence efficiency of Z-PDB pigments with respect to PDB molecules cannot be attributed to concentration effects such as dimerization, aggregation, bimolecular reactions or non-fluorescent traps excited through energy transfer. The absence of emitting dimers either inside the zeolite channels or on the zeolite external surface was confirmed by the analysis of the Z-PDB emission spectra, where, as it was already observed, the dimer-related shoulder at $560 \mathrm{~nm}$ in the emission spectrum of solvated PDB molecules disappears (see Fig. 2 and 3).

As for the physico-chemical properties of the channel environment, both the internal acidity and polarity were 
considered as the most probable factors that may affect the Z-PDB quantum yield. Emission spectra of PDB molecules dissolved in solvents with different polarities (i.e., dielectric constant $\varepsilon$ ) were measured. The following solvents were used: toluene $(\varepsilon=2.4)$, dichloromethane $(\varepsilon=9.1)$, acetone $(\varepsilon=20.7)$, and acetonitrile $(\varepsilon=37.5){ }^{[30]}$ The PDB concentration in the solution was kept as low as possible, in order to avoid aggregation. The absorption over $1 \mathrm{~cm}$ was set for all solutions at a value of 0.05 at $450 \mathrm{~nm}$. Since the integrated area under the emission spectra is constant, we can qualitatively conclude that the quantum yield is insensitive to the polarity of the molecule environment. Moreover, an ethanol-PDB solution was either acidified or basified with a few drops of hydrochloric acid solution $(1 \mathrm{M})$ or potassium hydroxide solution $(1 \mathrm{M})$, respectively. Again, the integrated area under the emission spectra remains almost unaffected. Therefore, neither the environmental polarity nor the acidity inside the zeolite channels can be considered as responsible for the low quantum yield of $\mathrm{Z}$ PDBs.

\section{Conclusions}

In conclusion, the quantum yield of fluorescent zeolite L nanoparticles loaded with perylene molecules was measured by a diffuse reflectance technique. In contrast to the most common relative quantum yield estimates based on the comparison with a fluorescent molecule in solution taken as reference, this technique intrinsically accounts for light scattering and self-absorption and directly yields reliable absolute quantum yield values.

We showed a decrease of the quantum yield of PDB molecules down to 0.4 once they are infiltrated in the zeolite channels. In particular, our measurements demonstrated that this can be attributed neither to the high concentration in the zeolite channels, nor to the dimer formation on the zeolite surface, nor to the physico-chemical properties of the channel environment.

Further phenomena may be considered to account for this unexpected strong decrease, such as i) the physical confinement of the PDB molecules into the channels or ii) the interaction between the inorganic host and the organic guest molecules. Once inside the channels, the PDB molecules are confined and a large steric interaction takes place between the inorganic host and the organic guest molecules. This interaction may affect the geometrical shape of the PDB molecules and, consequently, the transition dipole responsible for the emission process, thus limiting intrinsically the Z-PDB quantum yield. On the other hand, the interaction between the inorganic host and the organic
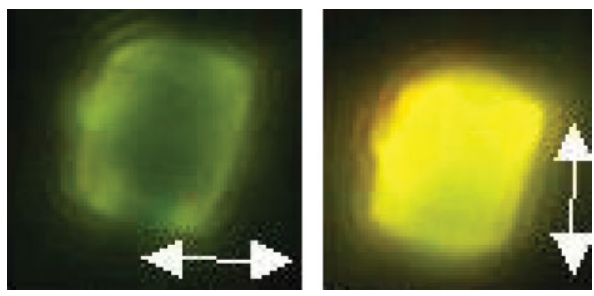

Figure 6. Fluorescence microscopy images of a $3-\mu \mathrm{m}$ zeolite $\mathrm{L}$ crystal loaded with $N, N^{\prime}$-dipentyl-3,4,9,10-perylenedicarboximide for different polarization orientations of the analyzer (white arrows). guest molecules may also contribute to lower the quantum yield value. In particular, as it is well known for perylenes, ${ }^{[31]}$ an electron transfer to the PDB molecule (e.g., either from the zeolite framework or from the cations inside the zeolite channels) may limit its fluorescence efficiency.

It is thus clear that a systematic study of the factors that affect the quantum yield of fluorescent zeolite pigments is necessary. Nevertheless, we observe that this goes beyond the objectives of this paper, which was intended to provide the zeolite community with the first experimental assessment of the quantum yield of zeolite nanoparticles. More generally, our results open up the subject of encapsulated dyes to a more thorough study of the effects of encapsulation on the dye quantum yield and provide researchers studying encapsulated functional nanopigments with a precise and reliable experimental technique for quantum yield measurements.

\section{Experimental}

Preparation of Z-PDB Pigments: Cylindrical zeolite L nanocrystals with an aspect ratio of 1:1 were synthesized using a hydrothermal process. Crystallization took place in an aqueous system containing the necessary reactants at high temperatures [32]. The synthesis parameters were set to obtain an average nanoparticle size in the order of $200 \mathrm{~nm}$. Size separation techniques, such as centrifugation or sedimentation, were used to minimize the presence of aggregates. A narrow size distribution around $200 \mathrm{~nm}$ was obtained.

PDB molecules were infiltrated into the zeolite channels using a gasphase method $[3,26]$. They are indeed rather large with respect to the diameter of the zeolite $L$ channels and an activation barrier has to be overcome to infiltrate them into the zeolite channels, that is, a temperature of $180^{\circ} \mathrm{C}$ is needed during the gas-phase loading. The actual infiltration of perylene molecules into the zeolite $L$ channels was assessed by polarized fluorescence microscopy measurements on $3-\mu \mathrm{m}$ zeolites synthesized and loaded with the same procedures as for the $200-\mathrm{nm}$ nanocrystals. A drop of a diluted dispersion was put on a microscopy glass slide and, after evaporation of the solvent, fluorescence microscopy measurements were made with a Zeiss Axiophot microscope. A mercury lamp with an excitation filter centered at $360 \mathrm{~nm}$ and a longpass emission filter of cut-on wavelength $420 \mathrm{~nm}$ were used. The fluorescence polarization was measured by using a linearly polarized analyzer. A strongly polarized anisotropy (see Fig. 6) was found as in Ref. [27], thus proving that the transition moments of the perylene molecules are aligned in the onedimensional channels of the zeolite crystals [33]. We note that, if the perylene molecules were adsorbed at the zeolite surface instead of inside the zeolite channels, no evidence of emission anisotropy would be found in the fluorescence images [27,33]. Nevertheless, we acknowledge that the physical-chemical mechanisms enabling the insertion of large molecules, such as PDB, into the channels of zeolite $L$ nanocrystals need to be further investigated.

After the infiltration of the PDB molecules by the gas-phase method, in order to test the zeolite loading stability, the Z-PDB pigments were successively rinsed with portions of fresh toluene and, after each washing step, the absorption and fluorescence of the washing solution were measured. The procedure was repeated until the detected signal was negligible, that is, until no PDB molecules could be found in the supernatant solution, thus obtaining Z-PDB pigments that were stable against any post-infiltration molecule leakage under these conditions. At the same time, the residual dye concentration in the washing solutions was evaluated measuring the solution absorption spectra by means of a standard UV-vis spectrophotometer. The difference between the initial dye content used in the gas-phase loading reaction and the residual amount of dye molecules in the washing solutions provided a first estimate of the loading level. 
In order to have a more reliable measurement of the loading efficiency, a known quantity of previously washed Z-PDB nanoparticles were treated with a hydrofluoric (HF) acid solution [34]. In a typical experiment, $3 \mathrm{~mL}$ of an $8 \%$-HF solution were added to a sonicated suspension of $11 \mathrm{mg}$ of ZPDB in $15 \mathrm{~mL}$ of ethanol in order to dissolve selectively the inorganic zeolite framework and to obtain a clear solution where the previously loaded PDB molecules were directly released. After having diluted $0.5 \mathrm{~mL}$ of the latter solution with $2.5 \mathrm{~mL}$ of ethanol, as above, the residual dye concentration was evaluated measuring the solution absorption spectrum by means of a standard UV-vis spectrophotometer. The exact amount of released PDB was obtained using the PDB extinction coefficient measured in reference solutions containing the same amount of HF-dissolved zeolites and a known amount of PDB molecules. Therefore, the exact quantity of PDB molecules loaded into the Z-PDB pigments (in $\mathrm{mg} \mathrm{g}^{-1}$ ) could be measured and the corresponding concentration in solution (in $\mathrm{mol} \mathrm{L}^{-1}$ ) was calculated. We remark that, if a homogeneous dye distribution inside the zeolite crystal is assumed, these quantities can be translated into the ZPDB loading efficiency, which is defined as the percentage of the available adsorption sites inside the zeolite channels that are occupied by the PDB molecules: when the loading efficiency is $100 \%$, the zeolite crystal is completely filled (i.e., all the available adsorption sites are occupied). In order to calculate this percentage, the average size of an encapsulated PDB molecule was roughly estimated to be in the order of 2.8 unit cells [35]. Note that the measured loading efficiencies are affected by a statistical experimental error in the order of $5 \%$.

Z-PDB pigments were fabricated with a quantity of loaded PDB molecules of $6.8,18.1,35.0$, and $38.9 \mathrm{mg}$ (PDB) $\mathrm{g}^{-1}$ (zeolite) (i.e., a concentration in solution of $0.03,0.09,0.17$, and $\left.0.19 \mathrm{~mol} \mathrm{~L}^{-1}\right)$ that corresponds to a loading efficiency of $12,32,62$, and $69 \%$, respectively (see Table 1).

Finally, note that, in order to measure their quantum yield, Z-PDB pigments were adsorbed into microcrystalline cellulose substrates using a solvent evaporation method under heating and reduced pressure (see below). In order to guarantee the pigment stability during this latter procedure (i.e., to prevent the neutral dye molecules from leaking out of the channels during the cellulose-pigment treatment), a plugging agent was used, which is a well known method to stabilize the zeolite pigments against different environmental conditions and treatments [36]. In particular, 2-(1,3-dioxo-1H-benzo[de]isoquinolin-2(3H)-yl)-1-methylpyridinium methyl sulfate cationic molecules were inserted into the nanocrystals via cation exchange from toluene using the phase transfer catalyst cryptofix $222(4,7,13,16,21,24$-hexaoxa-1,10-diazabicyclo[8.8.8]hexacosane). Since in zeolites $\mathrm{L}$ the channel environment is anionic and bares chargecompensation exchangeable cations, the 2-(1,3-dioxo-1H-benzo[de]isoquinolin-2 $(3 \mathrm{H})$-yl)-1-methylpyridinium cations are adsorbed at both channel entrances, thus blocking the channels and eventually preventing the PDB molecules from leaking. The modified Z-PDB pigments were then rinsed with ethanol in different environmental conditions: the fluorescence of the washing solution was measured and found negligible, thus confirming that no molecule leakage occurred. Moreover, comparing the fluorescence spectrum of bare and modified Z-PDB pigments, no difference was found in the emission intensity after the plugging procedure.

Preparation of PDB Solutions and Z-PDB Dispersions for Optical Characterization: PDB solutions and Z-PDB dispersions were prepared for the optical characterization of the fluorophors (i.e., steady-state excitation and emission spectra). Toluene (Acros, spectrophotometric grade), dichloromethane (Acros, for analysis), acetone (Carlo Erba, for analysis), and acetonitrile (Acros, for analysis) were used for the PDB solutions. The molecules were highly diluted $\left(10^{-6} \mathrm{M}\right)$ in order to minimize the dimer formation. Z-PDBs were dispersed in toluene $\left(5 \mathrm{mg} \mathrm{L}^{-1}\right)$, whose refractive index is almost equal to the refractive index of zeolites, thus minimizing the scattering effects. In particular, for the zeolite $L$ a refractive index of 1.48 at $589 \mathrm{~nm}$ was measured by means of an index matching solution composed of xylene (Acros, for analysis) and ethanol (Fluka, HPLC grad) $(82.3 / 17.7$ vol)

Preparation of Samples for Diffuse Reflectance Measurements: Microcrystalline cellulose (Fluka DS-O) was used to prepare the samples for diffuse reflectance measurements using the solvent evaporation method $[23,25,37]$. PDB solutions and Z-PDB dispersions were prepared in a 1:1 mixture of ethanol (Fluka, HPLC grade) and toluene (Acros, spectrophotometric grade) with different fluorophor concentrations. After the solvent evaporation, the fluorescent powders were placed in a powder holder suitable for measurements in the integrating sphere. We remark that the samples prepared for the same set of measurements were always processed together in order to guarantee the same optical properties for all substrates.

Steady-State Excitation/Emission Measurements: The steady-state excitation and emission spectra of dyes and zeolite pigments in solution were measured with a standard fluorimeter (Horiba Jobin-Yvon Fluoromax-3). In the case of the cellulose samples, a fluorimeter (Varian Cary Eclipse) equipped with an optical fiber was used.

Quantum Yield Measurement by a Diffuse Reflectance Technique [22-24]: The quantum yield of the investigated fluorophors was measured by a diffuse reflectance method [25].

In order to obtain the quantum yield value, the quantity $\phi_{\text {measured }}$ was defined as the ratio between the emitted $\left(I_{\text {Fluo }}\right)$ and the absorbed $\left(I_{\text {Abs }}\right)$ light intensities. $I_{\text {Fluo }}$ and $I_{\text {Abs }}$ can be calculated from the measured reflectances at the absorption wavelength $\lambda_{0}$ using the following equation [23]

$$
\begin{aligned}
\phi_{\text {measured }} & =\frac{I_{\text {Fluo }}}{I_{\text {Abs }}} \\
& =\frac{R_{\text {wof }}\left(\lambda_{0}\right)-R_{\mathrm{f}}\left(\lambda_{0}\right)}{\left(R_{\text {sub }}\left(\lambda_{0}\right)-R_{\mathrm{f}}\left(\lambda_{0}\right)\right)\left[f_{\mathrm{S}}-\frac{f_{\mathrm{S}, T}\left(1-R_{\mathrm{wof}}\left(\lambda_{0}\right)\right)}{\left(1-R_{\mathrm{f}}\left(\lambda_{0}\right)\right)}\right]}
\end{aligned}
$$

where $R_{\mathrm{f}}$ and $R_{\text {wof }}$ are the diffuse reflectances measured with and without a cut-on filter in front of the detector, respectively. $R_{\text {sub }}$ is the diffuse reflectance of the bare cellulose substrate. $f_{S}$ and $f_{S, T}$ are the correction factors that take into account the spectral response of the detector and a small residual transmission through the cut-on filter, i.e., [23]

$f_{S}=\frac{\int_{\lambda} I_{F}(\lambda) S(\lambda) d \lambda}{\int_{\lambda} I_{F}(\lambda) d \lambda}$

$f_{S, T}=\frac{\int_{\lambda} I_{\mathrm{F}}(\lambda) S(\lambda) T(\lambda) \mathrm{d} \lambda}{\int_{\lambda} I_{\mathrm{F}}(\lambda) \mathrm{d} \lambda}$

with $I_{F}(\lambda), S(\lambda)$, and $T(\lambda)$ corresponding to the steady-state fluorescence spectrum, the photodetector spectral efficiency, and the filter residual transmission spectrum, respectively. Note that an additional correction had to be introduced to take into account the large residual transmission through the cut-on filter [25], thus yielding the corrected quantum yield value $(\phi)$.

Finally, we observe that, since the Stokes shift is small (see Fig. 2), selfabsorption effects can affect the fluorescence spectrum $I_{\text {Fluo }}$ due to variations of the nanopigment concentration in the cellulose substrates. The Birk equation was then used to correct the $\phi$ value and to obtain the absolute quantum yield ( $\left.\phi_{\text {Cor }}\right)[23]$

$\phi_{\mathrm{Cor}}=\frac{\phi}{1-\alpha(1-\phi)}$

where the self-absorption probability $\alpha$ was calculated by dividing the integrated area of the steady-state emission spectrum by the corresponding 
area of the emission spectrum measured for a very low pigment concentration in the cellulose substrate (i.e., for almost negligible selfabsorption). To this purpose, the emission spectra were normalized at a wavelength where they are not affected by self-absorption (i.e., $\lambda=600 \mathrm{~nm}$ : see the insets of Fig. 3 ).

\section{Acknowledgements}

This work was supported by the Swiss Confederation Innovation Promotion Agency (Commission pour la Technologie et l'InnovationCTI) and the Swiss National Science Foundation (SNSF) through the CTI8184.1 EPRP-IW and the SNSF-200021-111856 projects, respectively.

Received: September 24, 2008 Published online: May 12, 2009

[1] M. A. Keane, Colloids Surf, A 1998, 138, 11.

[2] G. Calzaferri, S. Huber, H. Maas, C. Minkowski, Angew. Chem, Int. Ed. 2003, 42, 3732

[3] M. Pauchard, A. Devaux, G. Calzaferri, Chem. Eur. J. 2000, 6, 3456.

[4] D. Brühwiler, G. Calzaferri, Microporous Mesoporous Mater. 2004, 72, 1.

[5] S. Huber, A. Z. Ruiz, H. Li, G. Patrinoiu, C. Botta, G. Calzaferri, Inorg. Chim. Acta 2007, 360, 869.

[6] C. Sanchez, B. Lebeau, F. Chaput, J.-P. Boilot, Adv. Mater. 2003, 15, 1969.

[7] C. Sanchez, J. Mater. Chem. 2005, 15, 3559.

[8] C. Urlacher, C. Marco De Lucas, J. Mugnier, Synth. Met. 1997, 90, 199.

[9] Y.-G. Zhao, W.-K. Lu, Y. Ma, S.-S. Kim, S. T. Ho, T. J. Marks, Appl. Phys. Lett 2000, 77, 2961

[10] T. Hattori, Appl. Phys. Lett. 2000, 87, 3240

[11] M. L. Cano, V. Fornés, H. García, M. A. Miranda, J. Pérez-Prieto, J. Chem. Soc. Chem. Commun. 1995, 2477.

[12] H. García, S. García, J. Pérez-Prieto, J. C. Scaiano, J. Phys. Chem. 1996, 100, 18158.

[13] S. Suárez, A. Devaux, J. Bañuelos, O. Bossart, A. Kunzmann, G. Calzaferri, Adv. Funct. Mater. 2007, 17, 2298.

[14] J. Schneider, D. Fanter, M. Bauer, C. Schomburg, D. Wöhrle, G. SchulzEkloff, Microporous Mesoporous Mater. 2000, 39, 257.
[15] N. Tessler, V. Medvedev, M. Kases, S. Kan, U. Banin, Science 2002, 295 1506

[16] G. Calzaferri, S. Huber, A. Devaux, A. Z. Ruiz, H. Li, O. Bossart, L.-Q. Dieu, Proc. SPIE 2006, 6192, 619216-1.

[17] C. Sanchez, B. Lebeau, F. Chaput, J.-P. Boilot, Adv. Mater. 2003, 15, 1969.

[18] G. Calzaferri, D. Brühwiler, S. Megelski, M. Pfenniger, M. Pauchard, B. Hennessy, H. Maas, A. Devaux, U. Graf, Solid State Sci. 2000, 2, 421.

[19] J. R. Lakowicz, Principles of Fluorescence Spectroscopy, 2nd Ed., Kluwer Academic/Plenum Press, New York 1999.

[20] A. T. R. Williams, S. A. Winfield, J. N. Miller, Analyst 1983, 108, 1067.

[21] S. Dhami, A. J. de Mello, G. Rumbles, S. M. Bishop, D. Phillips, A. Beeby, Photochem. Photobiol. 1995, 61, 341.

[22] S. A. Ruetten, L. K. Thomas, J. Phys. Chem. B 1998, 102, 598.

[23] L. F. V. Ferreira, T. J. F. Branco, A. M. B. do Rego, ChemPhysChem 2004, 5, 1848.

[24] L. S. Rohwer, J. E. Martin, J. Lumin. 2005, 115, 77

[25] R. Ferrini, O. Nicolet, S. Huber, S. Chappellet, C. Lovey, J. Perrenoud, M. Pauchard, L. Zuppiroli, unpublished.

[26] S. Hashimoto, M. Hagiri, N. Matsubara, S. Tobita, Phys. Chem. Chem. Phys. 2001, 3, 5043.

[27] S. Huber, G. Calzaferri, ChemPhysChem 2004, 5, 239.

[28] A guide to recording fluorescence quantum yield can be found at http:// www.jobinyvon.com (accessed September 2008).

[29] A. Pardo, D. Reyman, J. M. L. Poyato, F. Medina, J. Lumin. 1992, 51, 269.

[30] J. A. Riddick, W. B. Bunger, Organic Solvents, Wiley, New York 1970.

[31] B. L. Su, D. Barthomeuf, M. Che, Microporous Mesoporous Mater. 2006, 90, 390.

[32] A. Z. Ruiz, D. Brühwiler, T. Ban, G. Calzaferri, Monatsh. Chem. 2005, 136, 77

[33] S. Megelski, A. Lieb, M. Pauchard, A. Drechsler, S. Glaus, C. Debus, A. J. Meixner, G. Calzaferri, J. Phys. Chem. B 2001, 105, 25.

[34] S. Huber, PhD Thesis, Uni Bern 2005, 134, Harvesting electronic excitation in host-guest materials for light energy conversion.

[35] J. T. Edward, J. Chem. Educ. 1970, 47, 261.

[36] H. Maas, A. Kathyr, G. Calzaferri, Microporous Mesoporous Mater. 2003, 65, 233.

[37] L. F. V. Ferreira, J. C. Netto- Ferreira, I. Khmelinskii, A. R. Garcia, S. M. B. Costa, Langmuir 1995, 11, 231. 